\title{
Influence of Patch-Size Variability on the Crystallization of Tetrahedral Patchy Particles
}

\author{
Flavio Romano, ${ }^{1,2, *}$ John Russo, ${ }^{1, \dagger}$ and Hajime Tanaka ${ }^{1, *}$ \\ ${ }^{1}$ Institute of Industrial Science, University of Tokyo, 4-6-1 Komaba, Meguro-ku, Tokyo 153-8505, Japan \\ ${ }^{2}$ Department of Chemistry, Physical and Theoretical Chemistry Laboratory, University of Oxford, \\ South Parks Road, Oxford OX1 3QZ, United Kingdom
}

(Received 5 July 2013; revised manuscript received 11 July 2014; published 25 September 2014)

\begin{abstract}
The understanding of disorder effects on crystallization is of fundamental and technological importance. It is well established by both theory and experiment that particle-size polydispersity hinders crystallization for isotropically interacting particles. Here, we address the effects of patch variability in a model for tetrahedral colloids, where polydispersity is introduced independently on the size, position, and strength of the attractive patches. Our simulations indicate that, unlike particle-size polydispersity, angular polydispersity has a minor impact on the crystallization properties of tetrahedral colloidal particles. Particles with angular polydispersity well within current experimental possibilities fully retain their crystallization properties, a result which should encourage the realization of colloidal crystals in experiment.
\end{abstract}

DOI: 10.1103/PhysRevLett.113.138303

PACS numbers: 82.70.Dd, 64.70.dg, 81.10.Aj, 81.16.Dn

The self-assembly of colloidal particles is a promising way to develop new materials with targeted properties. The possibility of tuning both the kinetics and the thermodynamics of these particles via the shape, surface, or solvent properties allows the realization of a broad spectrum of equilibrium and nonequilibrium structures, many of which are still unexplored [1]. Perhaps one of the most attractive features of colloidal particles is the possibility of tuning the properties of the surface with patches, i.e., functionalized spots whose number and geometrical arrangement largely determine the thermodynamic properties and the equilibrium ordered structures obtained at low effective temperatures [2]. The possibility of forming ordered structures on the micrometer scale by spontaneous crystallization of colloids is an attractive perspective in technological applications; indeed, particles with different degrees of anisotropy have been exploited in simulation and experiment to obtain crystals [3], quasicrystals [4], and, perhaps more importantly, open crystals [5-7]. Open colloidal crystals have a significant technological potential: the diamond crystal [8] (and even its amorphous phase [9]) has been shown to have photonic properties, and its theoretical study has been one of the driving forces of the field [10-12]. The approach of decorating particles with attractive spots is also experimentally proven $[13,14]$, and, in a milestone work, the experimental realization of a twodimensional (2D) open crystal of patchy particles has been reported [6].

One of the major challenges in the experimental realization of colloidal crystals is the control over the imperfections in particle fabrication. For example, it is a well established result that perfectly monodisperse hard spheres are very good crystal formers, but increasing particle-size polydispersity gradually favors local icosahedral structures that turn the system into a glass former [15-17]. The first experimental realizations of hard-sphere crystals required significant effort, due to the high degree of monodispersity required [18]. On the other hand, the effect of imperfections of patches for the crystallization of open crystals is yet unknown. Understanding this effect is very important in order to obtain spontaneously assembling crystals in experiment.

In this Letter, we report a numerical study of the effect of model fabrication imperfections on the phase diagram and on the crystallization process of a tetrahedral patchy particle model. We select the case of four patches since (i) it is the most technologically relevant and (ii) the crystalforming versus glass-forming behavior of such particles is determined precisely by the patch size [11]. Thus, it is an open question whether and to what extent crystallization is retained with polydisperse patches.

In our simulations, we use the Kern-Frenkel model for four-patch particles [19], where the centers of the patches are located on the vertices of a tetrahedron. The interaction potential $v(i, j)$ is written as

$$
v(i, j)=\left\{\begin{array}{cc}
\infty & \text { if } r_{i j}<\sigma \\
-\epsilon f\left(\hat{\mathbf{p}}_{i, k=1 \ldots 4}, \hat{\mathbf{p}}_{j, k=1 \ldots 4}\right) & \text { if } \sigma \leq r_{i j}<\sigma+\delta, \\
0 & \text { if } r_{i j} \geq \sigma+\delta
\end{array}\right.
$$

where $r_{i j}$ is the interparticle distance and the angular modulation function $f\left(\hat{\mathbf{p}}_{i, k=1 \ldots 4}, \hat{\mathbf{p}}_{j, k=1 \ldots 4}\right)$ is defined in terms of the unit vectors $\hat{\mathbf{p}}_{i, k=1 \ldots 4}$ that identify the centers of the patches (labeled $k$ ) on the surface of particle $i$. $f\left(\hat{\mathbf{p}}_{i, k=1 \ldots 4}, \hat{\mathbf{p}}_{j, k=1 \ldots 4}\right)$ is 0 unless there are some $k$ and $l$ such that $\hat{\mathbf{r}}_{i j} \cdot \hat{\mathbf{p}}_{i, k}<\cos \left(\theta_{i, k}\right)$ and $\hat{\mathbf{r}}_{j i} \cdot \hat{\mathbf{p}}_{j, l}<\cos \left(\theta_{j, l}\right)$. In other words, two particles feel an attraction of energy $\epsilon$ 


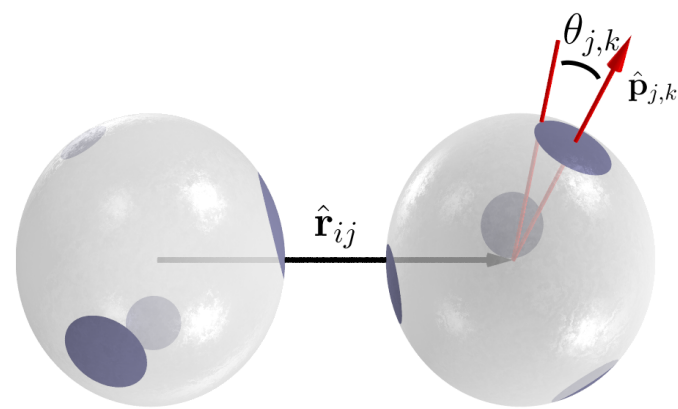

FIG. 1 (color online). Pictorial representation of two patchy particles in an interacting configuration. The particle's hard core is represented with a light gray sphere, whose surface is decorated with circular patches (dark Gray). Patch $k$ on the surface of particle $j$ is described by an angular extension $\theta_{j, k}$ and by the position vector $\hat{\mathbf{p}}_{i, k}$.

whenever they are within a distance $\sigma+\delta$ and the vector joining the centers of mass intersects a patch on the surface of each particle, as shown in Fig. 1. The patch size is identified by the parameter $\cos \left(\theta_{i, k}\right)$, and the radial interaction range is set to $\delta=0.24$. Reduced units are used throughout the Letter, with temperature $T$ measured in units of $k_{\mathrm{B}} / \epsilon$, pressure $P$ in units of $k_{\mathrm{B}} /\left(\epsilon \sigma^{-3}\right)$, and density $\rho$ in units of $\sigma^{-3}$. We run Monte Calro (MC) simulations in the $N P T$ ensemble. The presence of patch-size polydispersity effectively makes the particles distinguishable, considerably increasing the computational cost of the simulations. To ensure efficient sampling, we employ specialized MC moves [20].

For models with homogeneous patches, it has been shown that the interaction range $\delta$ controls the location of the liquid-gas critical point, but the metastability gap, measuring the crystal-forming ability of the liquid, does not change significantly with decreasing $\delta[21,22]$. In this Letter, we focus on the effects of disorder on the three main factors that control the crystallization behavior of tetrahedral patchy particles [21-23], i.e., size, position, and strength of the patches.

Size disorder is obtained by randomly choosing the patch width $\cos \left(\theta_{i, k}\right)$ from a uniform distribution. We introduce two types of patch-size polydispersity, allowing both intraparticle and interparticle inhomogeneity. In the first type, the patch size is independent for each patch: we label this distribution $p_{1}^{\text {ih }}$, with $\cos \left(\theta_{i, k}\right) \in[0.92,1]$. Here, the index $i=1, \ldots, N$ identifies the particle, and the index $k=1, \ldots, 4$ identifies the patch. In the second type, the four patches are the same for each particle but are independent among different particles. We used distributions with different average patch sizes but with the same width: $p_{2}^{\mathrm{h}}$ with $\cos (\theta) \in[0.92,0.96]$ and $\cos \left(\theta_{i, k}\right)$ the same for all $k$ and $p_{3}^{\mathrm{h}}$ with $\cos (\theta) \in[0.96,1]$ and $\cos \left(\theta_{i, k}\right)$ the same for all $k$. The widest patch-size range considered in our Letter, $p_{1}^{\text {ih }}$, is the largest possible that still retains the geometrical constraint of having only one possible bond per attractive patch [24]. Without this constraint, the geometric properties of the model are changed, and the extent to which the qualitative features of the phase diagram are preserved has yet to be investigated. Even within this limit, the patch-size distributions we consider here are very wide, up to \pm 10 degrees, where the average is around 15 degrees. As a comparison, we recall that the 2D colloidal crystal realized in [6] was obtained with a patch-size polydispersity of five degrees.

Positional disorder is obtained by perturbing the tetrahedral symmetry of the patch arrangement on the surface of the colloid. In spherical coordinates, each patch is displaced by a randomly extracted inclination angle $\psi$ from its original position. We consider three different $\psi$ distributions, $\psi \in\left[0, \psi_{\max }\right]$ with $\psi_{\max }=\pi / 36, \pi / 18, \pi / 12$, in which the displacement is comparable to the width of the patch interaction, $\psi_{\max } \approx \theta_{j k}$.

Energy disorder is implemented by altering the depth of the attractive interaction for each patch $\epsilon \pm \Delta \epsilon$, and then imposing additivity between particle interactions, $\epsilon_{i j}=\left(\epsilon_{i}+\epsilon_{j}\right) / 2$. We test three different disorder magnitudes, $\Delta \epsilon / \epsilon=0.1,0.2,0.3$, with patches on the same particles being different.

First, we investigate how the thermodynamics are changed by the three forms of disorder. We fix the pressure at a small positive value, $P=0.03$, safely above the critical pressure but still small enough to be relevant for colloidal systems. At this pressure, the low-temperature equilibrium phases are the open hexagonal and cubic diamond crystals. The coexistence temperature for each of the patch-size distributions considered is computed by Hamiltonian Gibbs-Duhem integration [25,26], using the coexistence temperature of the model with no patch-size polydispersity

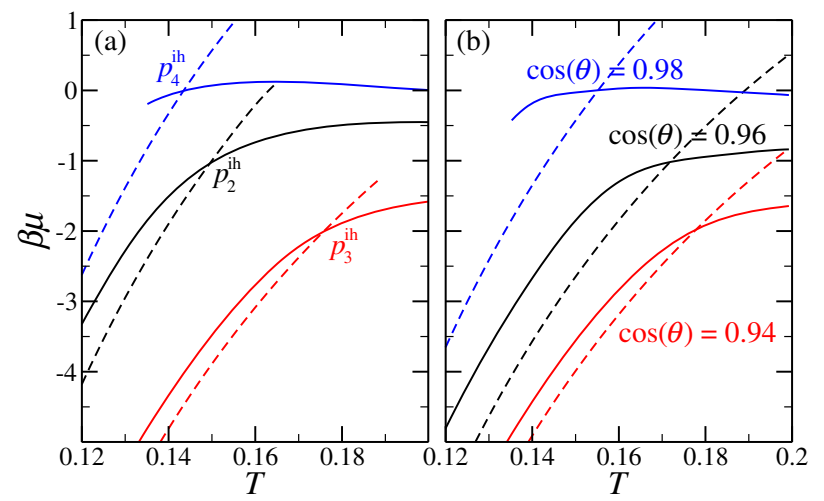

FIG. 2 (color online). Chemical potential of liquid (solid lines) and crystal (dashed lines) phases at pressure $P=0.03$ : (a) for systems with the three different patch-size distributions investigated and (b) for monodisperse systems with patch size corresponding to the average values of the patch-size distributions in (a). For clarity, absolute free-energy values have been shifted. A narrower average patch size consistently leads to a sharper increase in $\beta \Delta \mu$, in line with the behavior of the monodisperse particle studied in [23]. 
taken from [23]. In Fig. 2(b), we show the bulk chemical potentials $\beta \mu$ for the model, without disorder in the liquid and crystal phases, for three different amplitudes of the patch width. The liquid and crystal lines cross at the melting temperature, and the difference $\beta \Delta \mu$ below melting is the thermodynamic driving force, which quantifies the thermodynamic ease of crystallization for each system. The figure shows that the driving force, indeed, increases with decreasing patch width. In Fig. 2(a), we consider the effects of patch-size disorder for distributions with average size corresponding to the monodisperse case in Fig. 2(b). The comparison shows that the free energies of the two phases are only marginally affected by patch-size disorder, even with the widest distribution of patch sizes we consider. Although rather small, the destabilizing effect of the disordered patches is larger in the ordered crystal phase, indicating that the latter is less effective in accommodating the disorder. This is reflected in the slightly lowered coexistence temperatures with respect to monodisperse models. By comparing the different disorder distributions in Fig. 2(a), we see that the average size of patches retains a strong influence on the crystallization ability. Particles with wider patches [small $\left.\left\langle\cos \left(\theta_{k}\right)\right\rangle\right]$ have a small driving force for crystallization (defined as the difference between the chemical potential of the crystal and that of the liquid phase), which hardly increases with lowering temperature, indicating a poor crystal-forming ability. By lowering $\left\langle\cos \left(\theta_{k}\right)\right\rangle$, one effectively obtains a glass former [23]. Particles with narrower patches [large $\left.\left\langle\cos \left(\theta_{k}\right)\right\rangle\right]$, on the other hand, display a large driving force, sharply increasing with decreasing temperature, signaling an excellent crystal former. This shows that, even in the presence of strong disorder, the average patch size is still the key feature in the interaction potential that controls the crystallization ability in this model [23].

Interestingly, all models considered here display only a modest decrease of the melting temperature with increasing disorder. The introduction of disorder marginally favors the liquid phase for the following reason: disorder will produce patches that have a vanishing size, effectively altering the valence (coordination number) of the particles. This lowers the effective concentration of crystal-forming particles, requiring a lower temperature for nucleation. But this decrease is only about 5\%, showing that, together with the modest decrease of thermodynamic driving force, all the crystallization properties of the disorder-free model are retained in presence of size disorder. The amount of disorder can be arbitrarily large as long as the number of patches is four and that each patch is allowed to form only one bond. These are the only requirements for the nucleation of the diamond crystal phase considered here.

In Fig. 3(a), we focus on the effects of positional disorder. Since imperfect placement of the patches on the particles' surface alters their tetrahedral symmetry, this type of disorder can have a significant effect on their crystallization. We focus, here, on particles with patch size $\cos (\theta)=0.98$, i.e., on the good crystal formers (in the Supplemental Material [20] we show the same analysis, also, for systems with large patches). Figure 3(a) shows the thermodynamic driving force $\beta \Delta \mu$ as a function of the reduced temperature $\beta_{m}-\beta$ for systems with different amounts of positional disorder $\psi_{\max }$. Around the melting point $\left(\beta_{\mathrm{m}}-\beta=0\right)$, the thermodynamic driving force changes linearly, $\beta \Delta \mu=\Delta H\left(\beta_{\mathrm{m}}-\beta\right)$, where $\Delta H$ is the enthalpy change at melting. Figure 3(a) shows that $\Delta H$ does not change significantly with disorder until $\psi_{\max } \sim 20^{\circ}$, where the enthalpy difference between the liquid and the solid phase starts dropping significantly. For $\psi_{\max }<20^{\circ}$, the enthalpy difference between the liquid and solid phases does not change significantly with disorder, and so, the thermodynamic driving force increases considerably with supercooling. As we have shown in the
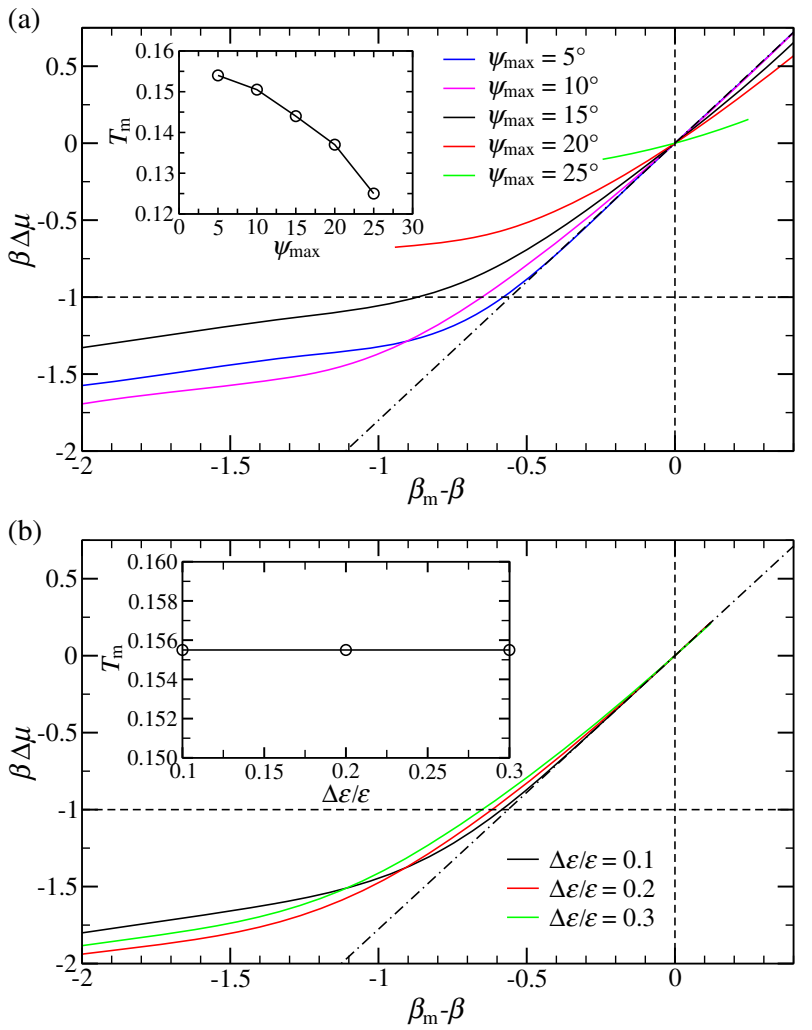

FIG. 3 (color online). Chemical potential difference between the liquid phase and the solid phase at pressure $P=0.03$ for systems with $\cos (\theta)=0.98$ as a function of the normalized temperature $\beta_{m}-\beta$ (with $\beta=1 / k_{\mathrm{B}} T$ ): (a) with positional disorder, (b) with energy disorder. Different lines represent systems with different degrees of disorder. The vertical dashed line represents the melting locus $\left(\beta_{m}-\beta=0\right)$. The horizontal dashed line indicates the thermodynamic driving force necessary to observe crystallization in the simulations $(\beta \Delta \mu \cong 1)$. The insets show the change of the melting temperature $\left(T_{m}\right)$ with positional disorder (a) and energy disorder (b). The dashed-dotted lines represent the thermodynamic driving force of crystallization given by $\beta \Delta \mu=\Delta H\left(\beta_{\mathrm{m}}-\beta\right)$. 
discussion of the patch-size disorder, the thermodynamic driving force is the factor that controls crystallization, and the amount of driving force necessary to observe crystallization depends almost entirely on the (average) size of the patches. For a system with $\cos (\theta)=0.98$, approximately $\Delta \mu \sim 1 k_{\mathrm{B}} T$ are needed to observe crystallization in simulation, and this limit is represented as a horizontal dashed line in Fig. 3(a). The figure shows that, for $\psi_{\max }<20^{\circ}$, crystallization in the diamond crystal is easily observed, while for $\psi_{\max } \gtrsim 20^{\circ}$, the system cannot reach the threshold of $\Delta \mu \sim 1 k_{\mathrm{B}} T$ regardless of the degree of supercooling. We note that, for the limiting case of $\psi_{\max }=20^{\circ}$, we could observe spontaneous crystallization from simulations, with the crystal containing many defects, or even comprising metastable phases different from the diamond. For both narrow and wide patches, the scenario is similar to what was found in Fig. 2 for the patch-width disorder: the major effect of positional disorder is to increase the free energy of the crystal phase, thus, lowering the melting temperature, shown in the inset of Fig. 3(a). The suppression of the melting point is modest for all disorders considered.

Figure 3(b) considers the effects of energy disorder, plotting the driving force $\beta \Delta \mu$ as a function of the reduced temperature $\beta_{m}-\beta$. Even for the highest degree of disorder $\Delta \epsilon / \epsilon=0.3$, the melting enthalpy (as represented by the slopes of the curves at $\beta_{m}-\beta=0$ ) and the increase in driving force with supercooling remain unchanged. Among all forms of disorder considered in this Letter, a polydisperse distribution of interaction energies has the least impact on the crystallization scenario. Simulations with $\Delta \epsilon / \epsilon \gtrsim 0.4$ still crystallize but with intervening fractionation; i.e., the crystal nucleus recruits all the particles with strong enough patches and then stops growing, leaving the particles with weaker patches in the fluid phase.

In the Supplemental Material [20], we also consider the change of the nucleation barriers as a function of size disorder, confirming that the features of the crystallization process of the monodisperse model are fully retained: the nucleation barrier goes down slowly with temperature in the wide patch case and sharply in the narrow patch case; we ascribe the majority of this effect to the different trends in the driving force shown in Fig. 2. We also investigated the coupling between patch-size disorder and the composition of crystals (see [20]). It is known that particle-size polydispersity induces demixing of hard spheres into crystals with different lattice sizes [15]. We find that patch-size disorder can introduce similar behavior, i.e., fractionation upon crystal nucleation.

To conclude, our focus was to explore the effects of angular disorder on the crystallization properties of patchy models, which are, nowadays, one of the major candidates for the experimental realization of three-dimensional colloidal crystals. Imperfections inevitably arising in the particle fabrication process will yield attractive patches that are polydisperse in size, shape, and strength. It is a common feeling among scientists that even small imperfections will favor the formation of disordered arrested states over ordered structures, a feeling borrowed from the well established result that particle-size polydispersity hinders crystallization of isotropic particles. On the contrary, we show that crystallization is very robust to imperfections in the patch size, position and interaction energy, as long as the average geometry allows bonds with the correct symmetry (tetrahedral in our case). More precisely, (i) patch-size polydispersity seems to have only a modest impact on crystallization as long as the patches can only be involved in a single bond at any given time, (ii) positional disorder of patches prevents crystallization only when it strongly perturbs tetrahedral symmetry $\left(\Psi_{\max }>20^{\circ}\right)$, and (iii) energy disorder has a negligible impact on crystallization. Our results suggest that a high degree of precision in the particle decoration is not needed to obtain long-range structures from patchy particles, and that currently available decoration techniques should have sufficient precision to reach the goal. Moreover, the robustness of the self-assembly behavior of patchy particles with respect to angular disorder further supports their use as a model system to understand the bulk properties of biological materials, like proteins and DNA, whose interactions are intrinsically anisotropic.

The authors thank F. Sciortino, J. Doye, and L. Rovigatti for helpful discussions. This study was partly supported by Grants-in-Aid for Scientific Research (S) and Specially Promoted Research from the Japan Society for the Promotion of Science (JSPS), the Aihara Project, the FIRST program from JSPS, initiated by the Council for Science and Technology Policy (CSTP). J. R. and H. T. acknowledge a JSPS Postdoctoral Fellowship for J. R. F. R. and H. T. acknowledge support from the JSPS for F. R.

F. R. and J. R. contributed equally to this work.

*flavio.romano@gmail.com

†russoj@iis.u-tokyo.ac.jp

tanaka@iis.u-tokyo.ac.jp

[1] S. C. Glotzer and M. J. Solomon, Nat. Mater. 6, 557 (2007).

[2] E. Bianchi, R. Blaak, and C. N. Likos, Phys. Chem. Chem. Phys. 13, 6397 (2011).

[3] M. E. Leunissen, C. G. Christova, A.-P. Hynninen, C. P. Royall, A. I. Campbell, A. Imhof, M. Dijkstra, R. Van Roij, and A. Van Blaaderen, Nature (London) 437, 235 (2005).

[4] A. Reinhardt, F. Romano, and J. P. K. Doye, Phys. Rev. Lett. 110, 255503 (2013).

[5] M. C. Rechtsman, F. H. Stillinger, and S. Torquato, Phys. Rev. E 75, 031403 (2007).

[6] Q. Chen, S. C. Bae, and S. Granick, Nature (London) 469 , 381 (2011).

[7] A. Jain, J. R. Errington, and T. M. Truskett, Soft Matter 9, 3866 (2013).

[8] M. Maldovan and E. L. Thomas, Nat. Mater. 3, 593 (2004). 
[9] K. Edagawa, S. Kanoko, and M. Notomi, Phys. Rev. Lett. 100, 013901 (2008).

[10] Z. Zhang, A. S. Keys, T. Chen, and S. C. Glotzer, Langmuir 21, 11547 (2005).

[11] F. Romano, E. Sanz, and F. Sciortino, J. Chem. Phys. 134, 174502 (2011).

[12] F. Romano and F. Sciortino, Nat. Commun. 3, 975 (2012).

[13] Y. Wang, Y. Wang, D. R. Breed, V. N. Manoharan, L. Feng, A. D. Hollingsworth, M. Weck, and D. J. Pine, Nature (London) 491, 51 (2012).

[14] A. B. Pawar and I. Kretzschmar, Macromol. Rapid Commun. 31, 150 (2010).

[15] P. Sollich and N. B. Wilding, Phys. Rev. Lett. 104, 118302 (2010).

[16] J. Russo and H. Tanaka, Sci. Rep. 2, 505 (2012).

[17] M. Leocmach, J. Russo, and H. Tanaka, J. Chem. Phys. 138, 12A536 (2013).
[18] C. P. Royall, W. C. K. Poon, and E. R. Weeks, Soft Matter 9, 17 (2013).

[19] N. Kern and D. Frenkel, J. Chem. Phys. 118, 9882 (2003).

[20] See Supplemental Material at http://link.aps.org/ supplemental/10.1103/PhysRevLett.113.138303 for the details.

[21] F. Romano, E. Sanz, and F. Sciortino, J. Phys. Chem. B 113, 15133 (2009).

[22] F. Romano, E. Sanz, and F. Sciortino, J. Chem. Phys. 132, 184501 (2010).

[23] I. Saika-Voivod, F. Romano, and F. Sciortino, J. Chem. Phys. 135, 124506 (2011)

[24] G. Foffi and F. Sciortino, J. Phys. Chem. B 111, 9702 (2007).

[25] M. Mehta and D. A. Kofke, Chem. Eng. Sci. 49, 2633 (1994).

[26] C. Vega, E. Sanz, J. Abascal, and E. Noya, J. Phys. Condens. Matter 20, 153101 (2008). 